\title{
Rapid detection of Cyfra 21-1 by optical-biosensing based on chemiluminescence immunoassay using bio-functionlized magnetic nanocomposites
}

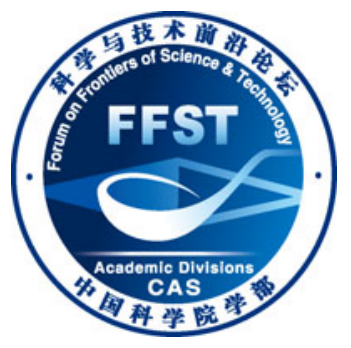

\author{
LUO JinPing $^{1}$, QU ShuXue ${ }^{1,2}$, LIU JunTao ${ }^{1}$, WANG Bin ${ }^{1,2}$ \& CAI XinXia ${ }^{1,2 *}$ \\ ${ }^{1}$ State Key Laboratory of Transducer Technology, Institute of Electronics, Chinese Academy of Sciences, Beijing 100190, China; \\ ${ }^{2}$ Graduate School of Chinese Academy of Sciences, Beijing 100190, China
}

Received October 23, 2012; accepted December 14, 2012; published online April 15, 2013

\begin{abstract}
This letter reports a chemiluminescene immunoassay method combined with immunomagnetic separation to rapidly detect Cyfra 21-1, in which bio-functionlized magnetic nanocomposites were used as mobile substrate for capturing and isolating the cyfra 21-1 proteins. After the captured Cyfra 21-1 further reacted with horseradish peroxidase-conjugated anti-Cyfra 21-1 antibody to form a sandwich immunocomplex, the chemiluminescence would be produced as a result of addition of the chemiluminescent substrate. A home-made optical biosensor was designed to detect the chemiluminescence instead of other large instruments. There is a good linear response between the chemiluminescence intensity and the concentration of Cyfra 21-1 in the range from 0.2 to 50 $\mathrm{ng} / \mathrm{mL}$. The whole detection process including incubation, washing and detection could be performed within 45 min. The proposed method offers a simple, noninvasive and reliable tool for detecting non-small cell lung cancer and has potential application for clinical testing.
\end{abstract}

Cyfra 21-1 antigen, bio-functionlized magnetic nanocomposites, chemiluminescence immunoassay

Citation: Luo J P, Qu S X, Liu J T, et al. Rapid detection of Cyfra 21-1 by optical-biosensing based on chemiluminescence immunoassay using bio-functionlized magnetic nanocomposites. Chin Sci Bull, 2013, 58: 2567-2569, doi: 10.1007/s11434-013-5736-z

Lung cancer is among the most common and lethal cancers worldwide. There were an estimated 1.61 million new cases with 1.38 million deaths in 2008 [1]. Non-small cell lung cancer (NSCLC) is one broad histological type of lung cancer and comprises approximately $85 \%$ of lung cancer cases. Tumor markers have been a complementary test in providing both an indication of response to therapy and disease progression or recurrence in cancer patients. Numerous tumor markers have been studied for potential use in NSCLC, such as carcinoembryonic antigen, squamous cell carcinoma, cytokeratin fragment 21-1 (Cyfra 21-1) and so on [2,3]. Of these, Cyfra 21-1 has been reported to be the most sensitive marker for NSCLC, and has considerable potential for early diagnostic of NSCLC, monitoring treatment response of patients and detecting recurrent disease after primary therapy.

*Corresponding author (email: xxcai@mail.ie.ac.cn)
The Cyfra 21-1 assay is a quantitative measurement of cytokeratin 19 fragments. The cytokeratins are a subfamily of intermediate filament-forming proteins containing at least 20 different polypeptides found in the intracytoplasmic cytoskeleton of epithelial tissue [4]. Cytokeratin 19 is especially expressed in simple epithelial cells and considered one of the main tumor markers for detection of NSCLC in the clinic. Up to date, several methods have been proposed to detect the Cyfra 21-1 concentration in body fluids, including radioimmunoassay [3,5], electrochemiluminsecent immunoassay [6], enzyme-linked immunosorbent assay [7,8], and chemiluminescent immunoassay (CLIA) [9,10]. The CLIA has gained increasing attentions due to the excellent properties, such as having high sensitivity, good specificity, simple equipment and wide linear range. However, limitations in the traditional CLIA method still remain because antibodies are usually immobilized on a solid sub- 
strate in air which will cause several problems. For example, the exposure of proteins to air seriously reduces their biological activity.

To resolve these problems, use of nanomaterials and nanotechnology has provided great opportunity for improving the traditional CLIA method. Here, we present our recent efforts to establish a CLIA method combined with immunomagnetic separation to detect Cyfra 21-1, in which bio-functionlized magnetic nanocomposites (BFMNs) were used as mobile substrate for capturing and isolating the Cyfra 21-1 proteins. When the captured Cyfra 21-1 further reacted with horseradish peroxidase (HRP)-conjugated anti-Cyfra 21-1 antibody to form a sandwich immunocomplex, the chemiluminescence would produced as a result of addition of the chemiluminescent (CL) substrate and detected by a home-made portable biosensor.

The BFMNs were fabricated in advance by anchoring the biotin-conjugated monoclonal anti-Cyfra 21-1 antibody onto streptavidin-coated superparamagnetic beads through a streptavidin-biotin bridge. The human Cyfra 21-1 antigen standard grade, HRP-conjugated murine monoclonal antibody and biotin-conjugated murine monoclonal antibody to Cyfra 21-1 used in the experiment were purchased from Xema-Medica, and the streptavidin-coated superparamagnetic beads M-280 with the size of the iron oxide less than $20 \mathrm{~nm}$ [11] were from Dynal Biotech. To our knowledge, the protein concentration could be qualified by the UV spectrophotometry. Since no other protein was introduced during the antibody-beads coupling process, the anti-Cyfra 21-1 antibody concentration could be calculated using the Lamber-Beer law and is proportional to the absorbency at $280 \mathrm{~nm}$ wavelength. Accordingly, the ratio of the immobilization antibodies in the fabrication of BFMNs was investigated by evaluated by measuring the absorbance of the initial antibody solution and the supernatant at $280 \mathrm{~nm}$ using a UV-1700 spectrophotometer (Shimadzu, Japan). The immobilization rate $R$ can be obtained as

$$
R=\left[\left(A_{0}-A_{1}\right) / A_{0}\right] \times 100 \%,
$$

where $A_{0}$ is the absorbency of the anti-Cyfra 21-1 antibody solution before immobilization, and $A_{1}$ is that of the unfixed anti-Cyfra 21-1 antibody in the supernatant after immobilization, respectively. It was calculated that the average immobility rate of capture antibody was about $92 \%$, indicating that the anti-Cyfra 21-1 capture antibody was effectively immobilized on the surface of superparamagnetic beads.

The home-made optical biosensor was designed to detect the chemiluminescence instead of other large instrument. It consists of two parts: a sensitive cell and an optical detector module. The sensitive cell was the key part to convert the concentration of Cyfra 21-1 to measuerable optical signals on the basis of the chemiluminescence immunoassay. The optical detector module was fabricated according to Wang et al. work [12], in which a photomultiplier tube with mode of H57155-20 and Field Programmable Gate Array were used to sensitively count the emitted photons. When the Cyfra 21-1 captured by the BFMNs and HRP-conjugated antibody reacts with CL substrate to produce chemiluminescence on the sensitive cell, the optical signals would be detected.

Cyfra 21-1 was detected by a sandwich-type immunoassay using two kinds of monoclonal antibodies which could recognize different epitopes of Cyfra 21-1. The schematic diagram of the analysis protocol is shown in Figure 1. There are two incubation steps and one wash step. The two incubation times are both no more than 15 min. Figure 2 shows the kinetics characteristics of CL reaction by detecting various concentrations of Cyfra 21-1 antigen standard. The CL reaction occurred immediately after addition of the substrate into the formed sandwich immunocomplex, and its intensity increased continuously. When the concentration of Cyfra $21-1$ is higher $(50 \mathrm{ng} / \mathrm{mL})$, the CL intensity increases rapidly in the former $4 \mathrm{~min}$ and tends to keep stable at latter time. When the concentration of Cyfra 21-1 is lower, it needs more time for CL intensity increasing to reach a plateau. Since too long triggering time for CL reaction was obviously disadvantageous to the improvement of assay speed, the optimal CL reaction time was chosen to be $6 \mathrm{~min}$. Therefore, the whole assay including incubation, washing and detection could be performed within $45 \mathrm{~min}$.

The relationship between Cyfra 21-1 concentrations and

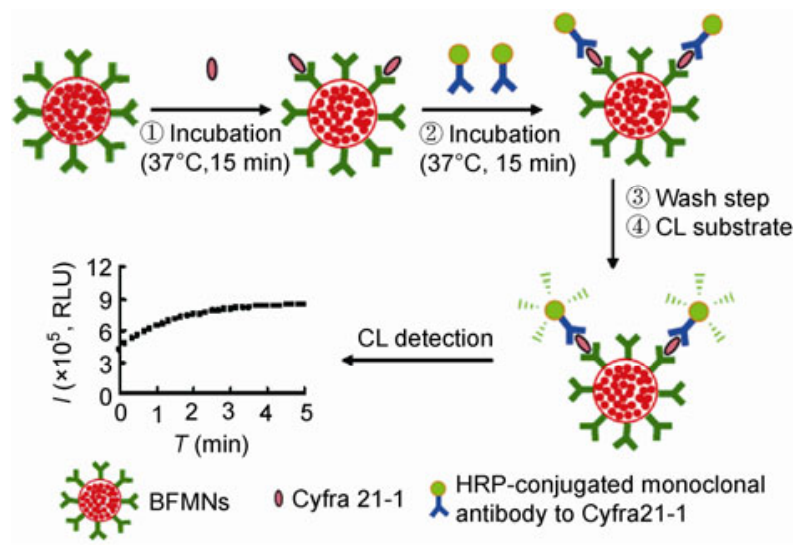

Figure 1 Schematic diagram of the analysis protocol.

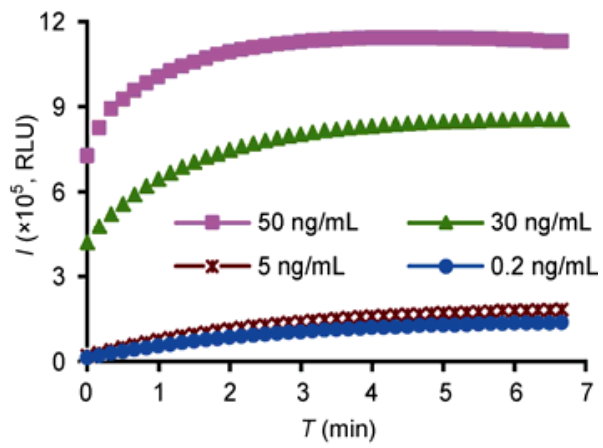

Figure 2 Kinetics curve for decreasing concentration of Cyfra 21-1 antigen $(50,30,5,0.2 \mathrm{ng} / \mathrm{mL})$. 
CL intensity was investigated by the home-made biosensor. As shown in Figure 3, there is a good linear response between the CL intensity and the concentration of Cyfra 21-1 in the concentration range from 0.2 to $50 \mathrm{ng} / \mathrm{mL}$. The linear regression equation for Cyfra 21-1 is

$$
I=0.218 C+0.549\left(R^{2}=0.939\right)
$$

where $I$ is the CL intensity and $C$ is the concentration of Cyfra 21-1. The detection limit of Cyfra 21-1 was 0.2 $\mathrm{ng} / \mathrm{mL}(S / N \geqslant 3)$.

In conclusion, this brief report provides a CLIA method combined with immunomagnetic separation to rapidly detect Cyfra 21-1, in which BFMNs were used as mobile substrate for capturing the Cyfra 21-1 proteins and the homemade optical biosensor was designed to detect the chemiluminescence instead of other large instrument. The whole

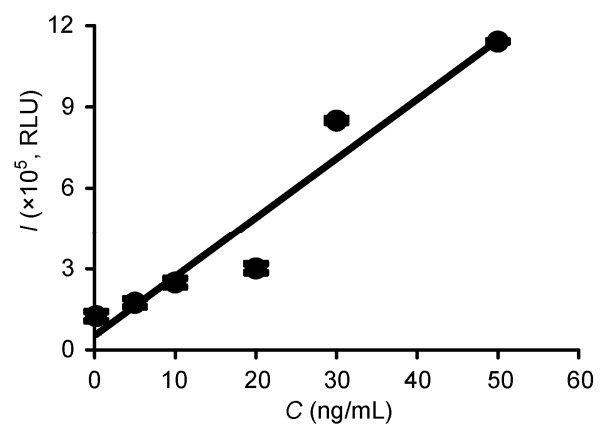

Figure 3 Calibration curve of CL intensity versus Cyfra 21-1 at the concentrations ranging from 0.2 to $50 \mathrm{ng} / \mathrm{mL}$. Each error bar was obtained from three measurements. detection process including incubation, washing, detection could be performed within $45 \mathrm{~min}$. The characteristics of detecting Cyfra 21-1 were primarily investigated to validate the proposed method. Our further work is to improve the detection precision and apply the proposed method for detecting clinical samples.

This work was supported by the National Natural Science Foundation of China (61125105, 61101048, 61002037, 61027001), the Major National Scientific Research Plan (2011CB933202), and the "Strategic Priority Research Program” of the Chinese Academy of Sciences (XDA06020101).

1 Ferlay J, Shin H R, Bray F, et al. Int J Cancer, 2010, 127: 2893-2917

William C C. Biomed Pharmacother, 2007, 61: 515-519

3 Hanagiri T, Sugaya M, Takenaka M, et al. Clin Lung Cancer, 2011, 74: 112-117

4 Barak V, Goike H, Panaretakis K W, et al. Clin Biochem, 2004, 37 : 529-540

5 Ceruse P, Rabilloud M, Charrie A, et al. Ann Otol Rhinol Laryngol, 2005, 114: 768-776

6 Deng Y F, Chen P, Lin Y Z, et al. J Laryngol Otol, 2003, 117: 190-194

7 Shimada H, Nabeya Y, Okazumi S, et al. J Am Coll Surg, 2003, 196: 573-578

8 Zhong L P, Zhou H G, Zhang C P, et al. Int J Oral Maxillofac Surg, 2007, 36: 230-234

9 Mizuguchi S, Nishiyama N, Lwata T, et al. Lung Cancer, 2007, 58: 369-375

10 Patel J L, Erickson J A, Roberts W L, et al. Clin Biochem, 2010, 41: 1449-1452

11 Fonnum G, Johansson C, Molteberg A. J Magn Magn Mater, 2005, 293: 41-47

12 Wang M X, Liu J T, Liu R P, et al. Chin J Sci Instrum, 2010, 31: 312-317

Open Access This article is distributed under the terms of the Creative Commons Attribution License which permits any use, distribution, and reproduction in any medium, provided the original author(s) and source are credited. 